\title{
APC Gold OA
}

Kyle Siler, Stefanie Haustein, Elise Smith, Vincent Larivière, Juan Pablo Alperin

\section{Source}

Table 1: Types and definitions of open access publishing.

Article published in a journal that charges publishing authors an APC; the article is freely available to all readers immediately. 